Journal of Patient-Centered

Volume 1

Issue 3 -- Cardio-Oncology

Article 5

8-13-2014

\title{
Cardiovascular Toxicity of Common Chemotherapy Drugs Used to Treat Breast Cancer: An Overview
}

Charles A. Bomzer

Follow this and additional works at: https://aah.org/jpcrr

Part of the Cardiology Commons, Chemicals and Drugs Commons, and the Oncology Commons

\section{Recommended Citation}

Bomzer CA. Cardiovascular toxicity of common chemotherapy drugs used to treat breast cancer: an overview. J Patient Cent Res Rev. 2014;1:133-136. doi: 10.17294/2330-0698.1027

Published quarterly by Midwest-based health system Advocate Aurora Health and indexed in PubMed Central, the Journal of Patient-Centered Research and Reviews (JPCRR) is an open access, peer-reviewed medical journal focused on disseminating scholarly works devoted to improving patient-centered care practices, health outcomes, and the patient experience. 


\section{Cardiovascular Toxicity of Common Chemotherapy Drugs Used to Treat Breast Cancer: An Overview}

Charles A. Bomzer, MD

Aurora Cancer Care, Aurora Health Care, Milwaukee, WI

\begin{abstract}
Treatment of breast cancer often exposes patients to many different drugs. Some of these drugs have toxic effects involving the cardiovascular system. This review provides an overview of the drugs most commonly used to treat breast cancer and their potential adverse impact on the cardiovascular system. ( $J$ Patient-Centered Res Rev. 2014; 1:133-136.)
\end{abstract}

\section{Keywords}

cardiovascular, breast cancer, chemotherapy

\section{Introduction}

The 5-year survival rate of women diagnosed with breast cancer between 1975 and 1977 was $75.1 \%$ and for women diagnosed from 2001 to 2007 was $90 \%$. This improvement in survival is primarily attributed to improvements in screening and the treatment of breast cancer. ${ }^{1,2}$ Along with the development of new drugs and improved survival has come a whole new understanding of short- and long-term effects of these treatments. It is estimated that in 2014 there will be 235,030 new cases of breast cancer $(232,670$ women and 2,360 men) diagnosed and 40,430 deaths from breast cancer (40,000 women and 430 men), ${ }^{2}$ thus making the impact of breast cancer treatment a meaningful concern. In this review we will highlight the common cardiovascular toxicities of drugs used to treat breast cancer and also mention some of the lesser known toxicities.

\section{Methods}

A search of the relevant literature using PubMed, Google and Bing was carried out. Search terms included: the drug name, cardiotoxicity, cardiovascular, toxicity, and cardiac. Online resources, such as the Centers for Disease Control and Prevention, National Cancer Institute, Food and Drug Administration and UpToDate ${ }^{\circledR}$ websites, also were utilized.

Correspondence: Charles A. Bomzer, MD, FACP, Aurora Cancer Care, 3003 W. Good Hope Road, Milwaukee, WI, 53209,

Phone: 414-352-6637, Fax: 414-247-4676,

Email: charles.bomzer@aurora.org
Studies were then reviewed for applicability to the topic and the data synthesized into a narrative form. Studies reporting cardiovascular toxicity of multiple drugs in combination were only included if those combinations were commonly used in the treatment of breast cancer. Side effects with a reported frequency of $<2 \%$ were generally excluded.

\section{Results}

\section{Anthracyclines and Anthracenediones}

The prototypical anthracycline drug in this class is doxorubicin. Other drugs in this class that are commonly used in breast cancer treatment include epirubicin and the sole approved anthracenedione, mitoxantrone. Less frequently used are liposomal doxorubicin and idarubicin. Although myocardial damage causing clinical heart failure or left ventricular dysfunction can be seen at low cumulative doses of these agents, the risk increases as the cumulative dose increases. In patients treated with doxorubicin, the estimated incidence of heart failure is 3-5\% at a cumulative dose of $400 \mathrm{mg} / \mathrm{m}^{2}, 7-26 \%$ at $550 \mathrm{mg} / \mathrm{m}^{2}$, and $18-48 \%$ at $700 \mathrm{mg} / \mathrm{m}^{2}$ of doxorubicin. The other anthracyclines and mitoxantrone have less cardiac toxicity, but each has its own unique cumulative cardiac dose limit. $^{3}$

The cardiac toxicity seen can be characterized as acute and nonacute, with authors variously subdividing the nonacute into subacute or early (onset less than 1 year after exposure) and late (onset more than 1 year after completion of treatment). ${ }^{3,4}$ Acute toxicity can include transient arrhythmias, pericarditis-myocarditis and reversible left ventricular dysfunction, which does not predict for later heart failure. ${ }^{4}$ The most worrisome complication is the nonacute toxicity of heart failure, which is usually irreversible. Consensus guidelines have been developed to manage heart failure due to anthracyclines and anthracenediones. ${ }^{5}$

Various strategies have been developed to try and reduce the risk of heart failure. These have included changing the schedule of administration, protective medications, and avoidance of concomitant administration of other potentially cardiotoxic medications. ${ }^{6}$ 


\section{Her2/neu Inhibitors}

Trastuzumab is the first of this class of drugs to be released to the market and was followed by lapatinib, pertuzumab and T-DM1. The incidence of significant cardiotoxicity (primarily manifest as heart failure) in trastuzumab-treated patients from various trials was $3-7 \%$ when the drug was used as a single agent and up to $28-34 \%$ when used in combinations, including with drugs like doxorubicin. Fortunately, this cardiotoxicity appears to respond to treatment and be reversible in a large number of patients on discontinuation of the agent. ${ }^{7}$ Pertuzumab has demonstrated no evidence of cardiotoxicity in studies to date. Lapatinib has a low likelihood of causing left ventricular dysfunction $(<2 \%) .{ }^{8}$ T-DM1 has a cardiotoxicity rate similar to lapatinib. ${ }^{9}$ Unique among this class of drugs, lapatinib causes an apparent dose-dependent prolongation of the QT interval and electrocardiographic (ECG) monitoring has been suggested (lapatinib [Tykerb] 2013 package insert, GlaxoSmithKline Inc., Research Triangle Park, NC). ${ }^{10}$

Recognizing the risks of cardiac dysfunction in patients treated with trastuzumab, investigators have established suggested monitoring intervals for echocardiograms. ${ }^{5}$ New data have emerged suggesting that serologic markers, in particular troponin I levels, may provide some predictive insights for this condition as well. ${ }^{11}$

\section{Fluoropyrimidines}

5-fluorouracil (5-FU) and its prodrug capecitabine have a different spectrum of cardiovascular toxicity than the aforementioned agents. Their cardiovascular toxicity is primarily related to ischemic cardiac events and is manifested by angina chest pain. ${ }^{12}$ For $5-\mathrm{FU}$, the reported incidence of cardiotoxicity varies from 1-68\% and usually appears within 2-5 days after the start of treatment. ${ }^{3}$ In the case of capecitabine, the incidence ranges from 3-9\% and onset of angina symptoms can occur from 3 hours to 4 days after starting treatment. ${ }^{10}$ Not unexpectedly, patients experiencing angina with 5-FU also may have the same symptoms with capecitabine.

Risk factors for cardiotoxicity include cardiac complications with another agent of this class, known coronary artery disease, continuous infusions, doses of 5-FU exceeding $800 \mathrm{mg} / \mathrm{m}^{2}$, prior mediastinal radiation, and concomitant administration of other chemotherapeutic agents. ${ }^{3,10}$ The condition should be treated in the same fashion as one treats ischemic cardiac disease, with the additional action of immediately stopping the fluoropyrimidine drug.

\section{Taxanes}

The microtubule-targeting drugs, paclitaxel and docetaxel, are both used to treat breast cancer. The incidence of left ventricular dysfunction differs for each, with docetaxel being less commonly implicated (2.3-8\%) than paclitaxel (5-15\%). However, the higher incidence of heart failure with paclitaxel is predominantly seen when it is combined with doxorubicin and is possibly due to the effects of paclitaxel on doxorubicin metabolism. ${ }^{3,13}$

The most frequently described cardiac effects of paclitaxel are bradycardia and heart block, ${ }^{14}$ but these are not seen with docetaxel. The arrhythmogenic effects of paclitaxel may be related to the vehicle in which it is diluted (Cremophor EL), which induces histamine release.

Docetaxel is associated with a distinct capillary leak syndrome that is caused by a capillary protein leakage and which can lead to peripheral edema, weight gain, pulmonary edema and hypoproteinemia. ${ }^{15}$ Steroid pretreatment has significantly reduced the incidence of this syndrome. ${ }^{16}$

\section{Other Microtubule-Targeting Drugs}

This class includes drugs such as the vinca alkaloids (vincristine, vinblastine and vinorelbine), eribulin and ixabepilone. In contemporary practice vincristine and vinblastine are infrequently used to treat breast cancer, but vinorelbine is a standard part of the armamentarium in the management of patients with metastatic breast cancer. Cardiotoxicity of vinca alkaloids is rare. Eribulin's most common side effects are neutropenia, fatigue and peripheral neuropathy, ${ }^{17}$ but an open-label ECG study demonstrated QT prolongation in a small number of patients. Consequently, the package insert recommends ECG monitoring if the drug is used in patients with congestive heart failure, bradyarrhythmias, electrolyte abnormalities, or who take drugs known to prolong the QT interval (eribulin [Halaven] 2013 package insert, Eisai Inc., Woodcliff Lake, NJ). Ixabepilone's most common nonhematologic toxicity is neuropathy. ${ }^{18-21}$ In one study, ${ }^{18} 7$ of 48 patients were reported to have "general" adverse cardiovascular effects (not specified). Using the National Cancer Institute Common Terminology Criteria for Adverse Events, 5 of these 7 patients were classified as grade 1 or 2, 2 were grade 3, and none were grade 4 . None of these cardiotoxicities were reported to cause discontinuation of therapy.

\section{Alkylating Agents}

The commonly used drugs in this class include cyclophosphamide and cisplatin (considered a bifunctional alkylator). Another formerly popular agent was thiotepa, but this is rarely used now. Cyclophosphamide has been reported to cause cardiotoxicity but only when used in high doses such as those used in regimens designed to prepare 
patients for bone marrow transplantation. ${ }^{10}$ The toxicity can include such conditions as asymptomatic pericardial effusions and heart failure. One study reported that 9 of 39 breast cancer patients prepared with 3 consecutive days of cyclophosphamide (2000 $\mathrm{mg} / \mathrm{m}^{2}$ per day) and thiotepa (200 $\mathrm{mm} / \mathrm{m}^{2}$ per day) experienced arrhythmias. Four of these occurred during stem cell infusion. ${ }^{22}$

Cisplatin is rarely associated with acute cardiovascular toxicities. ${ }^{10}$ Given the large amount of fluids usually administered with cisplatin infusions, careful attention must be paid to avoid hypervolemia and electrolyte disturbances.

\section{Discussion}

We are now entering an era in which, in many women, we are able to prolong survival enough to begin thinking of breast cancer as a chronic disease. This means that when treating these patients, we must constantly keep in mind both the short- and long-term implications of our treatment strategies and choices. Factors that increase the risk of developing cardiotoxicity (Table $1^{4,23,24}$ ) must be considered as well. In recognition of the need to predict and prevent the cardiovascular toxicity of chemotherapeutic agents, work has already begun on the development of protocols for monitoring and early detection of cardiotoxicity, with some guidelines already published. The guidelines are primarily empiric, representing expert opinion with some evidence to support them. Prudence would suggest the benefit of low-risk monitoring procedures, but costs also must be considered. Proposals for techniques and treatments to reduce the risk of cardiotoxicity have been postulated. . $^{4,53-25}$ These advances will foster greater collaboration between cardiologists and oncologists as we strive towards the common goal of healthier, more functional patients living longer with a better quality of life.

Table 1. Cardiovascular Risk Factors in Patients Receiving Chemotherapy $y^{4,25,27}$

\section{Extremes of age $(<15$ or $>65$ years $)$ \\ Preexisting cardiac risk factors (e.g. tobacco use, hypertension, coronary artery disease, dyslipidemia, diabetes mellitus, left ventricular dysfunction) \\ Chest/chest wall irradiation \\ Cumulative doxorubicin dose $>450 \mathrm{mg} / \mathrm{m}^{2}$ \\ Anthracyclines combined with chest/chest wall radiation}

\section{Conclusions}

The information contained in this paper provides a resource for clinicians involved in the care of patients with breast cancer. Breast cancer is one of the most common cancers that oncologists treat. Since a significant number of these patients will be exposed to chemotherapeutic agents at some time during the course of their treatment, it behooves all clinicians involved in the care of men and women with breast cancer to familiarize themselves with these drugs and their potential cardiovascular adverse effects. Although specific management recommendations are beyond the scope of this article, guidelines do exist and are available to the clinician to assist in patient management. Key to the successful management of potential cardiovascular toxicities is collaboration between oncologists and cardiologists.

\section{Conflicts of Interest}

Dr. Bomzer owns publicly traded stock in Merck Inc. and Pfizer Inc.

\section{References}

1. Berry DA, Cronin KA, Plevritis SK, et al. Effect of screening and adjuvant therapy on mortality from breast cancer. $N$ Engl $\mathrm{J} \mathrm{Med.}$ 2005;353:1784-92.

2. Siegel R, Ma J, Zou Z, Jemal, A. Cancer statistics, 2014. CA Cancer J Clin. 2014;64:9-29.

3. Bonita R, Pradhan R. Cardiovascular toxicities of cancer chemotherapy. Semin Oncol. 2013;40:156-67.

4. Steingart RM, Yadav N, Manrique C, Carver JR, Liu J. Cancer survivorship: cardiotoxic therapy in the adult cancer patient; cardiac outcomes with recommendations for patient management. Semin Oncol. 2013;40:690-708

5. Curigliano G, Cardinale D, Suter $T$, et al. Cardiovascular toxicity induced by chemotherapy, targeted agents and radiotherapy: ESMO Clinical Practice Guidelines. Ann Oncol. 2012;23 Suppl 7:vii155-66.

6. Ng R, Better N, Green M. Anticancer agents and cardiotoxicity. Semin Oncol. 2006;33:2-14.

7. Ewer SM, Ewer MS. Cardiotoxicity profile of trastuzumab. Drug Saf. 2008;31:459-67.

8. Perez EA, Koehler M, Byrne J, Preston AJ, Rappold E, Ewer MS. Cardiac safety of lapatinib: pooled analysis of 3689 patients enrolled in clinical trials. Mayo Clin Proc. 2008;83:679-86.

9. Sendur MA, Aksoy S, Altundag K. Cardiotoxicity of novel HER2targeted therapies. Curr Med Res Opin. 2013;29:1015-24.

10. Yeh ET, Bickford CL. Cardiovascular complications of cancer therapy: incidence, pathogenesis, diagnosis, and management. $J \mathrm{Am}$ Coll Cardiol. 2009;53:2231-47.

11. Cardinale D, Colombo A, Torrisi R, et al. Trastuzumab-induced cardiotoxicity: clinical and prognostic implications of troponin I evaluation. J Clin Oncol. 2010;28:3910-6.

12. Saif MW, Shah MM, Shah AR. Fluoropyrimidine-associated cardiotoxicity: revisited. Expert Opin Drug Saf. 2009;8:191-202.

13. Gehl J, Boesgaard M, Paaske T, Vittrup Jensen B, Dombernowsky P. Combined doxorubicin and paclitaxel in advanced breast cancer: effective and cardiotoxic. Ann Oncol. 1996;7:687-93.

14. Rowinsky EK, McGuire WP, Guarnieri T, Fisherman JS, Christian MC, Donehower RC. Cardiac disturbances during the administration of taxol. J Clin Oncol. 1991;9:1704-12.

15. Semb KA, Aamdal S, Oian P. Capillary protein leak syndrome appears to explain fluid retention in cancer patients who receive docetaxel treatment. J Clin Oncol. 1998;16:3426-32.

16. Hainsworth JD. Practical aspects of weekly docetaxel administration schedules. Oncologist. 2004;9:538-45.

17. Preston JN, Trivedi MV. Eribulin: a novel cytotoxic chemotherapy agent. Ann Pharmacother. 2012;46:802-11. 
18. Thomas E, Tabernero J, Fornier M, et al. Phase II clinical trial of ixabepilone (BMS-247550), an epothilone B analog, in patients with taxane-resistant metastatic breast cancer. J Clin Oncol. 2007;25: 3399-406.

19. Perez EA, Lerzo G, Pivot X, et al. Efficacy and safety of ixabepilone (BMS-247550) in a phase II study of patients with advanced breast cancer resistant to an anthracycline, a taxane, and capecitabine. J Clin Oncol. 2007;25:3407-14.

20. Roché H, Yelle L, Cognetti F, et al. Phase II clinical trial of ixabepilone (BMS-247550), an epothilone B analog, as first-line therapy in patients with metastatic breast cancer previously treated with anthracycline chemotherapy. J Clin Oncol. 2007;25:3415-20.

21. Denduluri N, Low JA, Lee JJ, et al. Phase II trial of ixabepilone, an epothilone B analog, in patients with metastatic breast cancer previously untreated with taxanes. J Clin Oncol. 2007;25:3421-7.
22. Ando M, Yokozawa T, Sawada J, et al. Cardiac conduction abnormalities in patients with breast cancer undergoing highdose chemotherapy and stem cell transplantation. Bone Marrow Transplant. 2000;25:185-9.

23. Bovelli D, Plataniotis G, Roila F; ESMO Guidelines Working Group. Cardiotoxicity of chemotherapeutic agents and radiotherapy-related heart disease: ESMO Clinical Practice Guidelines. Ann Oncol. 2010;21 Suppl 5):v277-82.

24. Carver JR, Szalda D, Ky B. Asymptomatic cardiac toxicity in longterm cancer survivors: defining the population and recommendations for surveillance. Semin Oncol. 2013;40:229-38.

25. Cardinale D, Bacchiani G, Beggiato M, Colombo A, Cipolla CM. Strategies to prevent and treat cardiovascular risk in cancer patients. Semin Oncol. 2013;40:186-98.

(C) 2014 Aurora Health Care, Inc. 